\title{
Carbapenemase-Producing Elizabethkingia Meningoseptica from Healthy Pigs Associated with Colistin Use in Spain
}

\author{
Pedro Miguela-Villoldo ${ }^{1,2}$, Marta Hernández ${ }^{3,4}$, Miguel Á. Moreno ${ }^{1,2, *}$, \\ David Rodríguez-Lázaro ${ }^{4}$, Alberto Quesada ${ }^{5,6}$, Lucas Domínguez ${ }^{1,2}$ and María Ugarte-Ruiz ${ }^{1}$ (iD \\ 1 VISAVET Health Surveillance Centre, Universidad Complutense, Madrid 28040, Spain \\ 2 Departamento de Sanidad Animal, Facultad de Veterinaria, Universidad Complutense, 28040 Madrid, Spain \\ 3 Laboratorio de Biología Molecular y Microbiología, Instituto Tecnológico Agrario de Castilla y León, \\ 47071 Valladolid, Spain \\ 4 Área de Microbiología, Departamento de Biotecnología y Ciencia de los Alimentos, Universidad de Burgos, \\ 09001 Burgos, Spain \\ 5 Departamento de Bioquímica, Biología Molecular y Genética, Facultad de Veterinaria, Universidad de \\ Extremadura, 10003 Cáceres, Spain \\ 6 INBIO G+C, Universidad de Extremadura, 10003 Cáceres, Spain \\ * Correspondence: mamoreno@ucm.es; Tel.: +34-913-943-705; Fax: +34-913-943-795
}

Received: 20 August 2019; Accepted: 6 September 2019; Published: 11 September 2019

\begin{abstract}
Carbapenems are considered last-resort antimicrobials, especially for treating infections involving multidrug-resistant Gram-negative bacteria. In recent years, extended-spectrum $\beta$-lactamase (ESBL) and carbapenemase-producing Gram-negative bacteria have become widespread in hospitals, community settings, and the environment, reducing the range of effective therapeutic alternatives. The use of colistin to treat infection caused by these multi-drug bacteria may favour the selection and persistence of carbapenem-resistant bacteria. In this study, it is described, for the first time to our knowledge, a carbapenemase-producing isolate of Elizabethkingia meningoseptica from healthy pigs in Spain. The isolate we report was recovered during a study to detect colistin-resistant bacteria from faecal samples of healthy food-production animals using a chromogenic selective medium. Unexpectedly, we found an isolate of Elizabethkingia meningoseptica with high Minimum Inhibitory Concentration (MIC) values for several antibiotics tested. Molecular analysis did not show any $m c r$ family genes related with colistin resistance, but two carbapenemase genes, bla $a_{\mathrm{B}-12 \_1}$ and $b l a_{\mathrm{GOB}-17 \_1}$, were detected. This finding in healthy animals could suggest that colistin may favour the selection and persistence of carbapenem-resistant bacteria.
\end{abstract}

Keywords: carbapenemase; colistin; Elizabethkingia meningoseptica; Antimicrobial resistance

Carbapenems are considered last-resort antimicrobials, especially for treating infections involving multidrug-resistant Gram-negative bacteria [1]. In recent years, extended-spectrum $\beta$-lactamase (ESBL) and carbapenemase-producing Gram-negative bacteria have become widespread in hospitals, community settings and the environment, reducing the range of effective therapeutic alternatives [1]. The most common carbapenemases include metallo- $\beta$-lactamases (MBLs), Klebsiella pneumoniae carbapenemases (KPC) and class D oxacillinases [1]. Since the first detection in 2011 in Germany, various European countries have reported carbapenemase-producing Escherichia coli and Salmonella in food-producing animals [2]. In Spain, although described in pets and synanthropic animals [3,4], carbapenemase-producing Escherichia coli has not been detected in food-producing animals. To our knowledge, this is the first report of a carbapenemase-producing isolate of Elizabethkingia meningoseptica from healthy pigs in Spain. 
Elizabethkingia species (genus formerly known as Chryseobacterium) are Gram-negative bacilli commonly found in freshwater, saltwater, soil and hospital environments [5]. Infections caused by Elizabethkingia spp., particularly E. meningoseptica, have been reported and are associated with a high fatality risk, particularly in immunocompromised patients [5].

The isolate we report was recovered during a study to detect colistin-resistant bacteria [6]. Antimicrobial chemotherapy, particularly colistin, has been the treatment of first choice to control Gram-negative bacteria in pig production, habitually used as a prophylactic [7]. Currently, the use of colistin is only allowed to treat infections caused by multidrug-resistant bacteria and metaphylaxis [8]. To carry out this study, we used a chromogenic selective medium. Identification involved mass spectrometry using a Bruker Daltonics UltrafleXtrem MALDI TOF/TOF instrument (Bruker Daltonics, Bremen, Germany) and Whole Genome Sequencing (WGS) to confirm the species and to further characterize the isolate. Elisabethkingia meningoseptica isolate was, $99 \%$ identity (accession number: NZ_CP016378.1).

Phenotypic characterization of antimicrobial susceptibility was performed. Minimal inhibitory concentrations (MICs) were determined using a two-fold broth microdilution reference method, according to ISO 20776-1:2006 [9]. Due to broad resistance of the Elisabethkingia genus and the diverse therapeutic alternatives described in literature for infections associated with this bacterium, two different antimicrobial panels (EUVSEC and EUST) were used, covering different antimicrobial families commonly used for Gram-negative and Gram-positive bacteria (Trek Diagnostic Systems, US). The isolate showed a carbapenemase-producing profile: high MIC values for carbapenems (meropenem MIC $=16 \mathrm{mg} / \mathrm{L} ;$ imipenem MIC $=16 \mathrm{mg} / \mathrm{L} ;$ ertapenem MIC $>2 \mathrm{mg} / \mathrm{L})$. This isolate also presented high MIC values for colistin (MIC > $16 \mathrm{mg} / \mathrm{L}$ ), ampicillin (MIC $>64 \mathrm{mg} / \mathrm{L}$ ), gentamicin (MIC $=32 \mathrm{mg} / \mathrm{L}$ ), ceftazidime (MIC $=128 \mathrm{mg} / \mathrm{L})$, and cefotaxime $(\mathrm{MIC}=32 \mathrm{mg} / \mathrm{L})$. These results agree with reports that Elizabethkingia species are resistant to polymyxins, tetracycline and, especially, $\beta$-lactams, including carbapenems, due to the production of chromosomal MBLs. Two types of MBLs have been identified in E. meningoseptica (BlaB and GOB), and both types of MBLs can be found in the same strain. BlaB-12 and GOB-17 have been reported in a clinical human isolate in Korea [5]. We tested antimicrobials usually employed to treat Gram-positive infections, notably vancomycin and rifampicin which have been used to treat human infections caused by E. meningoseptica [5]. Our isolate had a vancomycin MIC of $8 \mu \mathrm{g} / \mathrm{mg}$ and rifampicin MIC of $0.25 \mu \mathrm{g} / \mathrm{mg}$.

We determined the whole genome sequence of the isolate. Sequencing libraries were prepared using the Nextera XT kit and sequenced on a MiSeq (Illumina, San Diego, CA, USA) using v3 reagents with $2 \times 300$ cycles. The resistome of the draft genome was searched against ResFinder database [10] revealing two carbapenemase-encoding genes, $b l a_{\mathrm{B}-12 \_1}$ (98\% identity) and $b l a_{\mathrm{GOB}-17 \_1}(100 \%$ identity). No other antimicrobial resistance genes were detected. The putative presence of plasmids was evaluated by BLASTn searches against the Plasmid Finder database [11]. No plasmids were found, indicating that the carbapenemase genes $b l a_{\mathrm{B}-12_{-} 1}$ and $b a_{\mathrm{GOB}-17_{-} 1}$ were on the chromosome, consistent with what is commonly found for MBLs in this species [5]. Further analysis to confirm this finding are underway.

The presence of ESBL- and carbapenemase-producing E. coli in livestock has been associated with colistin resistance $[1,12-14]$ and we observed in a previous study that Colistin-resistant $E$. coli in pigs has also been linked to colistin sales [6]. Colistin has been the treatment of first choice to control these bacteria in pig production and it has habitually used as a prophylactic approach as well [7]. Over the past decade, resistance to colistin has emerged becoming a public health problem mainly due to the spread of plasmid mobile genes in the $\mathrm{mor}$ family. Thus, these colistin resistance genes could co-occur with ESBL and/or carbapenemase genes in the same isolate, promoting the co-selection. Because of that fact, these data suggest that colistin use may favour the selection and persistence of carbapenem-resistant bacteria. Further studies are needed to assess whether indeed colistin use in food animals, especially in pigs, is associated with a risk of co-selection of colistin, and more surprisingly, carbapenem resistance. 
Author Contributions: Conceptualization, P.M.-V., M.A.M., L.D. and M.U.-R.; Methodology, P.M.-V., M.H., D.R.-L.; Software, M.H., D.R.-L. and A.Q.; Validation, M.H., D.R.-L. and A.Q.; Formal Analysis, P.M.-V., M.H., M.A.M., D.R.-L., A.Q., L.D. and M.U.-R.; Investigation, P.M.-V., M.A.M., L.D. and M.U.-R.; Resources, P.M.-V., M.A.M., L.D. and M.U.-R.; Data Curation, P.M.-V., M.A.M., L.D. and M.U.-R.; Writing-Original Draft Preparation, P.M.-V., M.A.M., L.D. and M.U.-R.; Writing-Review \& Editing, P.M.-V., M.H., M.A.M., D.R.L., A.Q., L.D. and M.U.-R.; Visualization, P.M.-V., M.H., M.A.M., D.R.-L., A.Q., L.D. and M.U.-R.; Supervision, P.M.-V., M.H., M.A.M., D.R.-L., A.Q., L.D. and M.U.R.; Project Administration, P.M.-V., M.A.M., L.D. and M.U.-R.; Funding Acquisition, P.M.-V., M.A.M., L.D. and M.U.-R.

Funding: This research was funded by the Spanish Ministry of Economy, Industry, and Competitiveness (AGL2016-74882-C3), the Spanish Ministry of Agriculture, Fishing, and Food, and the Autonomous Community of Madrid (S2013/ABI-2747). Pedro Miguela-Villoldo was supported by the FPI Program (BES-2017-080264) from the Spanish Ministry of Science, Innovation and Universities.

Acknowledgments: The authors wish to thank the technicians María García, Estefanía Rivero, Nisrin Maasoumi and Estefanía Martínez for their excellent technical assistance at the Foodborne Zoonoses and Antibiotic Resistance Unit.

Conflicts of Interest: The authors declare no conflict of interest.

\section{References}

1. Dandachi, I.; Chabou, S.; Daoud, Z.; Rolain, J.M. Prevalence and Emergence of Extended-Spectrum Cephalosporin-, Carbapenem- and Colistin-Resistant Gram Negative Bacteria of Animal Origin in the Mediterranean Basin. Front. Microbiol. 2018, 9, 2299. [CrossRef] [PubMed]

2. The European Union summary report on antimicrobial resistance in zoonotic and indicator bacteria from humans, animals and food in 2016. EFSA J. 2018, 16, e05182.

3. Gonzalez-Torralba, A.; Oteo, J.; Asenjo, A.; Bautista, V.; Fuentes, E.; Alos, J.I. Survey of Carbapenemase-Producing Enterobacteriaceae in Companion Dogs in Madrid, Spain. Antimicrob. Agents Chemother. 2016, 60, 2499-2501. [CrossRef] [PubMed]

4. Vergara, A.; Pitart, C.; Montalvo, T.; Roca, I.; Sabate, S.; Hurtado, J.C.; Planell, R.; Marco, F.; Ramírez, B.; Peracho, V. Prevalence of Extended-Spectrum-beta-Lactamase- and/or Carbapenemase-Producing Escherichia coli Isolated from Yellow-Legged Gulls from Barcelona, Spain. Antimicrob. Agents Chemother. 2017, 61, e02071-16. [PubMed]

5. Ceyhan, M.; Celik, M. Elizabethkingia meningosepticum (Chryseobacterium meningosepticum) Infections in Children. Int. J. Pediatr. 2011, 2011, 215237. [CrossRef] [PubMed]

6. Miguela-Villoldo, P.; Hernández, M.; Moreno, M.A.; Rodríguez-Lázaro, D.; Quesada, A.; Domínguez, L.; Ugarte-Ruiz, M. National colistin sales versus colistin resistance in Spanish pig production. Res. Vet. Sci. 2019, 123, 141-143. [CrossRef] [PubMed]

7. Moreno, M.A. Survey of quantitative antimicrobial consumption per production stage in farrow-to-finish pig farms in Spain. Vet. Rec. Open 2014, 1, e000002. [CrossRef] [PubMed]

8. European Medicines Agency. Updated Advice on the Use of Colistin Products in Animals within the European Union: Development of Resistance and Possible Impact on Human and Animal Health; European Medicines Agency: Amsterdam, The Netherlands, 2016.

9. International Organization for Standarization (ISO). CLinical Laboratory Testing and In Vitro Diagnostic Test Systems-Susceptibility Testing of Infectious Agents and Evaluation of Performance of Antimicrobial Susceptibility Test Devices-Part 1: Reference Method for Testing the In Vitro Activity of Antimicrobial Agents against Rapidly Growing Aerobic Bacteria Involved in Infectious Diseases; ISO 20776-1:2006; International Organization for Standarization: Geneva, Switzerland, 2006.

10. Zankari, E.; Hasman, H.; Cosentino, S.; Vestergaard, M.; Rasmussen, S.; Lund, O.; Aarestrup, F.M.; Larsen, M.V. Identification of acquired antimicrobial resistance genes. J. Antimicrob. Chemother. 2012, 67, 2640-2644. [CrossRef] [PubMed]

11. Carattoli, A.; Zankari, E.; Garcia-Fernandez, A.; Voldby Larsen, M.; Lund, O.; Villa, L.; Aarestrup, F.M.; Hasman, H. In silico detection and typing of plasmids using PlasmidFinder and plasmid multilocus sequence typing. Antimicrob. Agents Chemother. 2014, 58, 3895-3903. [CrossRef] [PubMed]

12. Garcia, V.; Garcia-Menino, I.; Mora, A.; Flament-Simon, S.C.; Diaz-Jimenez, D.; Blanco, J.E.; Alonso, M.P.; Blanco, J. Co-occurrence of mcr-1, mcr-4 and mcr-5 genes in multidrug-resistant ST10 Enterotoxigenic and 
Shiga toxin-producing Escherichia coli in Spain (2006-2017). Int. J. Antimicrob. Agents 2018, 52, 104-108. [CrossRef] [PubMed]

13. Barragan-Prada, H.; Ruiz-Hueso, P.; Tedim, A.P.; Gonzalez-Candelas, F.; Galan, J.C.; Canton, R.; Morosini, M.I. Emergence and dissemination of colistin-resistant Klebsiella pneumoniae isolates expressing OXA-48 plus CTX-M-15 in patients not previously treated with colistin in a Spanish university hospital. Diagn. Microbiol. Infect. Dis. 2019, 93, 147-153. [CrossRef] [PubMed]

14. Pulss, S.; Semmler, T.; Prenger-Berninghoff, E.; Bauerfeind, R.; Ewers, C. First report of an Escherichia coli strain from swine carrying an OXA-181 carbapenemase and the colistin resistance determinant MCR-1. Int. J. Antimicrob. Agents 2017, 50, 232-236. [CrossRef] [PubMed]

(C) 2019 by the authors. Licensee MDPI, Basel, Switzerland. This article is an open access article distributed under the terms and conditions of the Creative Commons Attribution (CC BY) license (http://creativecommons.org/licenses/by/4.0/). 